\title{
Recent Insights Into Cyberchondria
}

\author{
Vladan Starcevic $^{1,2}($ DD $)$ David Berle ${ }^{3,4} \cdot$ Sandra Arnáez ${ }^{5}$
}

Published online: 27 August 2020

(C) Springer Science+Business Media, LLC, part of Springer Nature 2020

\begin{abstract}
Purpose of Review The construct of cyberchondria was introduced relatively recently. This article aims to review the conceptualization, theoretical basis and correlates of cyberchondria, as well as its prevention and management.

Recent Findings Although there is no consensus, most definitions of cyberchondria emphasize online health research associated with heightened distress or anxiety. The two theoretical models of cyberchondria involve reassurance seeking and specific metacognitive beliefs. Cyberchondria has relationships with health anxiety, problematic Internet use and symptoms of obsessive-compulsive disorder, with public health implications pertaining to functional impairment and altered healthcare utilization. Suggestions about prevention and management of cyberchondria have been put forward, but not tested yet.

Summary Research interest in cyberchondria has steadily increased. It is uncertain whether cyberchondria can be considered a distinct entity. Future research should aim to clarify the conceptual status of cyberchondria, quantify its impact and develop evidence-based approaches for a better control of cyberchondria.
\end{abstract}

Keywords Cyberchondria $\cdot$ Online health research $\cdot$ Reassurance seeking $\cdot$ Health anxiety $\cdot$ Problematic Internet use Compulsivity

\section{Introduction}

The concept of cyberchondria has an interesting 20-year history. There is some controversy as to where the term first appeared and to whom it can be attributed: the main contenders are a 1999 Wall Street Journal article and a 2001 article published in The Independent, but others also occasionally pop up [1]. Regardless of who coined the term

This article is part of the Topical collection on Anxiety Disorders

Vladan Starcevic

vladan.starcevic@sydney.edu.au

1 Discipline of Psychiatry, Faculty of Medicine and Health, Sydney Medical School, Nepean Clinical School, University of Sydney, Sydney, NSW, Australia

2 Department of Psychiatry, Nepean Hospital, PO Box 63, Penrith, NSW 2751, Australia

3 Graduate School of Health, Discipline of Clinical Psychology, University of Technology Sydney, Ultimo, NSW, Australia

4 University of New South Wales, School of Psychiatry, Sydney, NSW, Australia

5 Facultad de Psicología, Departamento de Personalidad, Evaluación y Tratamientos Psicológicos, University of Valencia, Valencia, Spain cyberchondria, it is certain that its creator was not a mental health clinician or researcher. Cyberchondria was introduced in the context of the early days of the Internet, at the time of an increasing interest in the "negative side" of this new and revolutionary information and communication medium. There was much fascination with the newspaper stories depicting cases of "Internet addiction" and other novel Internet-related afflictions, often with a sensationalist slant. Therefore, it is not surprising that these terms were promoted by journalists, which may also help explain why cyberchondria was not taken seriously by clinicians and researchers for about a decade.

Two landmark articles in 2009 by White and Horvitz [2, 3] served as a catalyst for further academic investigations in the area. These Microsoft researchers conducted a large-scale study of the factors that led to an escalation of health worries during online searches for health-related information and in the quest for self-diagnosis on the Internet. Over the subsequent years, academic researchers started paying more attention to cyberchondria, and both theoretical articles on the subject and empirical studies of cyberchondria started to appear. In 2014, the first instrument for the assessment of cyberchondria, the Cyberchondria Severity Scale (CSS) [4], was published, which gave a significant impetus to research activity and scholarly work. This is reflected in many research, 
review and theoretical articles published on cyberchondria and related topics since then.

The aim of the present article is to review selected key issues about cyberchondria. These include the conceptualization and definition, theoretical perspectives, relationships with other constructs, impairment, cost, public health implications and prevention and management aspects. Assessment issues will also be addressed briefly, as will the topic of cyberchondria during the COVID-19 pandemic.

\section{Conceptualization of Cyberchondria}

Defining cyberchondria has proved to be challenging. There are two main approaches to defining it. The first emphasizes the link with health anxiety and conceptualizes cyberchondria as an excessive and/or repetitive pattern of online health research (OHR) that is associated with an increase in health anxiety or distress [5]. The second approach is broader and suggests that cyberchondria is syndrome-like and multidimensional in that it reflects both "anxiety and an element of compulsiveness" and includes several components: repetitive and time-consuming OHR, negative emotional states (e.g., anxiety) or distress associated with OHR, interruption of other activities as a result of OHR and consulting a physician in response to an increased distress or anxiety, usually to seek reassurance [4]. With its construal of cyberchondria as a syndrome-like entity, the second approach also paves the way for potentially considering cyberchondria as a distinct construct. Indeed, the results of a study using a network analysis approach were consistent with a syndrome-like conceptualization and a relatively independent status for cyberchondria [6••]. However, this may be partly due to some circularity, as this study relied on the instrument (CSS) developed on the basis of the multidimensional conceptualization of cyberchondria. Interestingly, the network analysis also showed that features of cyberchondria were interrelated, with no feature clearly emerging as more central to the construct of cyberchondria - a finding that calls for further investigation of the structure of the cyberchondria construct. No conceptualization of cyberchondria includes a disease or diseases that drive OHR. This is likely to be a consequence of the shifting focus of OHR between the different individuals and perhaps even within a single individual over time.

A consensus about the meaning and scope of cyberchondria is still missing. Some authors reject the term because of the "confusion" surrounding its various meanings $[7 \bullet \bullet]$. A recent systematic review $[8 \bullet \bullet]$ reported that definitions of cyberchondria in various publications most commonly mentioned an "increase in anxiety" (89.8\% of articles), followed by "compulsive or repetitive behaviour" (66.1\%). One element that is common to all definitions is a behavioural pattern of OHR, regardless of how it is further characterized (e.g., as excessive, time-consuming, problematic, repetitive or compulsive). The second element that unifies various definitions is a recognition that OHR is associated with a negative emotional state, such as distress, anxiety or health anxiety.

Recently, an "extended" or "working definition" of cyberchondria has been proposed [8・•]. It includes several components: (1) pattern of excessive OHR; (2) characterization of OHR as "compulsive", "hard to resist" and serving the purpose of seeking reassurance; (3) short-lived relief with OHR because "anxiety or distress usually worsens.... and persists afterwards"; (4) prioritization of OHR over other interests or activities and its continuation or escalation despite its negative consequences. While this definition is certainly comprehensive, it is unclear whether all the proposed components of cyberchondria need to be present. Also, it remains to be ascertained whether some of these components are more important than others, i.e., whether they always need to be present, whereas others may be optional. Furthermore, this definition implicitly gives cyberchondria a disorder-like or diagnosis-like status and emphasizes the similarities with behavioural addictions. Although the relationship between cyberchondria and problematic Internet use (PIU) as a potential behavioural addiction has been demonstrated [6••, 9-13], conceptualizing cyberchondria as a behavioural addiction seems premature because it reflects a more severe variant of the condition. Finally, cyberchondria may be better defined as a dimensional construct, on a continuum from a mild to severe behavioural and psychopathological pattern.

Further research on cyberchondria crucially depends on the adoption of a consensus-based definition and conceptualization. Given the present state of understanding and knowledge, the most useful definition may be the one that relies on the description of the relevant behaviours and phenomena, without suggesting potential psychopathological mechanisms and relationships. From this perspective, a succinct and effective definition of cyberchondria would include OHR as a behaviour that is associated with distress or anxiety; interference with functioning might be added to emphasize clinical and public health significance.

\section{Theoretical Perspectives on Cyberchondria}

The first theoretical account of cyberchondria was the reassurance-seeking model [1], which posits that individuals with high levels of health anxiety engage in OHR to be reassured about their health concerns. As the outcome of reassurance seeking on the Internet is largely unpredictable because of the nature of the Internet, some people are reassured by what they find online, whereas others are not. Those who fail to experience reassurance or feel only partially reassured and are therefore more anxious continue with OHR in an effort to find reassurance. OHR also persists owing to the 
factors that reinforce it, including a need for definitive explanations, information overload, uncertainty inherent to an online search process and questionable trustworthiness of the sources of online information. In short, OHR in cyberchondria is regarded as a maladaptive coping with health anxiety that creates a vicious cycle and perpetuates itself. This pattern corresponds to what was later called "problematic OHR" $[7 \bullet \bullet]$.

According to the metacognitive model of cyberchondria $[11,14,15]$, OHR and its negative effects, including increased anxiety and distress, are related to certain metacognitive beliefs. These beliefs may be about the usefulness of the Internet for coping with health-related distress and anxiety (positive metacognitive beliefs) or about the loss of control over OHR and a sense that OHR is detrimental (negative metacognitive beliefs). If the negative metacognitive beliefs are more prominent, a perceived threat then comes from OHR itself so that OHR is experienced as distressing, compulsive and out of control-a pattern labelled as "compulsive OHR" [7••]. While problematic OHR and cyberchondria based on reassurance seeking is more clearly and more strongly related to health anxiety, compulsive OHR and cyberchondria related to negative metacognitive beliefs may have a stronger link with PIU. Therefore, there may be potentially two types of "pathological OHR": problematic OHR and compulsive OHR [7••].

The idea about the two types of pathological OHR and, by extension, two "subtypes" of cyberchondria, needs to be elaborated and tested. At present, it is not clear whether these two patterns are relatively separate and if so, what implications that may have for understanding, prevention and management of cyberchondria. In fact, it was suggested that cyberchondria might be a "unitary construct" and include both problematic OHR and compulsive OHR, although these components may be present in different proportions in different individuals and perhaps at different times in the same individuals [16]. If so, a better understanding of cyberchondria at an individual level calls for a meticulous formulation that specifies the possible mechanisms and reinforcing factors.

One aspect of cyberchondria that needs further theoretical development is its compulsive nature. This is because of the relationships with PIU and obsessive-compulsive disorder (OCD) and the need to understand and target the person's motivation for continued OHR despite its negative consequences and previous experience of it being ineffectual with regard to reassurance. The negative metacognitive beliefs, especially those about the loss of control over OHR, may contribute to persistent $\mathrm{OHR}$. Other potential reasons are more clearly related to compulsivity, with an expectation of further negative consequences if OHR were to stop. Such consequences may pertain to a fear of missing out on some information deemed to be relevant and important for one's health concerns. These considerations might help in better intertwining the various features and aspects of cyberchondria: OHR, health anxiety (or health-related distress), reassurance seeking and compulsivity.

The conceptual independence of cyberchondria is another important issue. As already noted, the network analysis study has suggested that cyberchondria might be relatively specific and distinct from all related constructs [6••]. This finding needs to be replicated before cyberchondria can be conceptualized as an entity in its own right - a difficult task because there are no clear guidelines about the minimum requirements for any psychopathological entity to be considered separate and distinct from related conditions.

\section{Relationships Between Cyberchondria and Other Constructs}

The concept of cyberchondria was developed on the basis of its hypothesized relationships with health anxiety and hypochondriasis. Many studies [11, 12, 14, 17-26] have confirmed moderate to strong correlations between cyberchondria and health anxiety ( $r$ s ranging from 0.48 to 0.68 , depending on the instrument used for the assessment of health anxiety). Only one study [13] found this correlation to be relatively weak $(r=0.23)$. A recent systematic review and metaanalysis $[27 \bullet \bullet$ confirmed a positive and strong correlation between cyberchondria and health anxiety $(r=0.62,95 \%$ $\mathrm{CI}=0.52-0.71, p<0.0001)$. The direction of any causal relationship between these two constructs remains uncertain because most research has used a cross-sectional design. Although heightened levels of health anxiety precede cyberchondria and lead to it, which is the usual assumption, excessive OHR may induce health anxiety or increase its levels in vulnerable individuals. One longitudinal study has demonstrated the latter pathway [28].

In addition to health anxiety, PIU and symptoms of OCD also have important relationships with cyberchondria, although these have not been studied so extensively. The correlations between cyberchondria and PIU have been robust and ranged between 0.43 and 0.59 , depending on the instrument used for measuring PIU [11-13]. These correlations reflect features that are common to both cyberchondria and PIU: excessive participation in online activities, diminished control over them so that online engagement cannot decrease and persistence of online activities despite their negative consequences. Moreover, individuals with cyberchondria were found to be more likely to take part in other types of problematic online activities [9].

The correlations between cyberchondria and OCD symptoms have similarly been robust and ranged from 0.38 to 0.56 $[14,17,21,22]$ for the total scores on the Dimensional Obsessive-Compulsive Scale. When the correlations were calculated for scores on the subscales of the Dimensional 
Obsessive-Compulsive Scale, they were lower $(r \mathrm{~s}=0.21-$ $0.44)[22,29]$, as were the correlations with scores on the subscales of the Maudsley Obsessive-Compulsive Inventory $(r \mathrm{~s}=0.27-0.40)[30]$. These findings may suggest that the specific symptom subtypes of OCD have different relationships with cyberchondria, but this has not been reported consistently. Compulsivity and reassurance-seeking behaviour that characterize both cyberchondria and OCD may account for the relationship between cyberchondria and OCD symptoms.

Intolerance of uncertainty is a construct related to cyberchondria on theoretical grounds [1]. Moreover, both health anxiety and OCD, constructs associated with cyberchondria, have independent and strong relationships with intolerance of uncertainty. Not surprisingly, studies have repeatedly shown moderate to strong correlations between cyberchondria and intolerance of uncertainty. These correlations were stronger with inhibitory intolerance of uncertainty $(r \mathrm{~s}=0.31-0.52)$ than with prospective intolerance of uncertainty $(r \mathrm{~s}=0.24-0.44)[11,14,18,19,30,31]$. Inhibitory intolerance of uncertainty refers to a tendency to inhibit behaviour and feel paralyzed in response to uncertainty, whereas prospective intolerance of uncertainty denotes an anxious anticipation of the future and need for greater predictability of future events. Some studies suggested that intolerance of uncertainty might be a specific risk factor for cyberchondria [18, $19,30,31]$. Other research reported a nonspecific association between intolerance of uncertainty and cyberchondria [11, 14], which is in line with the transdiagnostic nature of intolerance of uncertainty. Consequently, intolerance of uncertainty may relate to cyberchondria indirectly, for example, via their common link with health anxiety [31]. Regardless of the exact nature of this relationship, it appears to be important and should be taken into account in an effort to better understand cyberchondria.

The relationships between cyberchondria and other constructs were also investigated by means of a network analysis approach $[6 \bullet \cdot]$. Using this method, the authors demonstrated that although cyberchondria was strongly related to both PIU and health anxiety, it was closer to the former than to the latter.

In view of these findings, it can be concluded that cyberchondria may overlap with health anxiety/hypochondriasis, PIU and OCD, despite some research suggesting that this overlap is relatively small [6••]. Other studies have found that cyberchondria and health anxiety may represent distinct constructs [29, 32], although they are closely related, overlap and similarly pertain to various feared illnesses. Therefore, it is simplistic to consider cyberchondria only a modern counterpart to hypochondriasis. Likewise, cyberchondria should not be portrayed only as a form of PIU or as an entity solely related to OCD. The ways in which these constructs interact may be more complex and future studies are expected to shed more light on their interactions.

\section{Impairment, Cost and Public Health Implications of Cyberchondria}

Cyberchondria refers to a time-consuming activity over which there is little or no perceived control. Individuals with cyberchondria are therefore prone to neglecting or deprioritizing their duties and activities at home, work or learning environments. Relationships and social life may be adversely affected as well. Research confirms an association between cyberchondria and functional impairment, even when controlling for the effect of health anxiety [32]. However, a better understanding of the impact of cyberchondria on various domains of functioning calls for more research.

Cyberchondria may also have effects on seeking and receiving healthcare, which has public health implications. Thus, cyberchondria may drive help- and treatment-seeking behaviour as a result of the anxiety induced by excessive OHR. This may lead people with cyberchondria to ask for more frequent consultations with their primary care physicians and other doctors or to request various medical investigations. Indeed, cyberchondria has been associated with an increased healthcare utilization, measured by the number of visits to various healthcare professionals [20].

Some individuals may attempt to cope with cyberchondriainduced distress by avoiding contact with healthcare professionals. Such avoidance may lead to a failure to seek appropriate healthcare, with further negative consequences. Another problematic aspect of cyberchondria is a negative impact on the patient-physician relationship [4, 10], for example, via tendency of some individuals with cyberchondria to challenge physicians about diagnosis and treatment. Research findings in this domain have been conflicting, partly because of the failure to distinguish between normal and pathological OHR. Thus, some studies reported arguments and disputes between patients and physicians occurring in the context of OHR $[33,34]$, whereas others found the opposite pattern, i.e. an improved communication and interaction [35-37]. The frequency of adversarial interactions between individuals with cyberchondria and physicians and consequently poor treatment adherence remains unknown.

The cost of cyberchondria and the harm associated with it have not been calculated. Likewise, economic burden of cyberchondria and its public health significance remain to be ascertained more precisely. Given the observations and preliminary findings about functional impairment associated with cyberchondria and its effects on health-related behaviours, healthcare utilization and communication and relationships with healthcare professionals, there are reasons to believe that the personal and societal impacts of cyberchondria is significant. 


\section{Assessment of Cyberchondria}

Several instruments for the assessment of cyberchondria have been developed, but the CSS [4] has been by far the most frequently used tool. The CSS is based on a multidimensional conceptualization of cyberchondria [4], and in addition to the total score, it provides scores on 5 subscales, which represent each cyberchondria dimension: compulsion (interference with other activities), distress, excessiveness, reassurance and mistrust of medical professionals. The original version of the CSS consists of 33 items, but its shorter and modified versions (with 30, 15 and 12 items) have also been used. The CSS has been translated into several languages.

A recent review of the instruments developed for the assessment of cyberchondria [38•] commented that much of our current understanding of cyberchondria is derived from research in which the CSS was used and suggested that the dominant status of the CSS among the measures of cyberchondria resulted from its very good to excellent psychometric properties and its reliance on a solid theoretical framework. However, there is room for improvement of the CSS, and it deserves further research scrutiny, especially with regard to its factor structure, divergent validity, test-retest reliability, use in clinical populations and scoring system. Moreover, questions have been raised about the construct validity of the CSS, i.e. the unresolved issue of what this instrument measures, whether it can distinguish between normal and pathological OHR and whether it taps all the relevant components of cyberchondria [7••]. With regard to the latter, it has been suggested that a comprehensive cyberchondria instrument might also need to include items that assess the perception of OHR, especially in terms of its controllability, intrusiveness and notion about the amount of the needed health information $[7 \cdot \bullet]$.

Finally, the use of the CSS implies a direct or indirect endorsement of the theoretical framework on which it is based [38•], while there remains a lack of consensus about the definition of cyberchondria. Consequently, in addition to the need for refinement of the CSS, there is also a need for the multidimensional conceptualization of cyberchondria to be tested.

\section{Cyberchondria During the COVID-19 Pandemic}

The COVID-19 (coronavirus) pandemic has been an unprecedented event of the digital era, considering the disruption that it has caused in all areas of life worldwide. The pandemic has been a fertile soil for cyberchondria because of the fear induced by COVID-19 ("coronaphobia", "COVID-19 anxiety"); the uncertainty associated with the fact that COVID19 is a novel disease for which the world was poorly prepared; the abundance of online, unverified and constantly updated information on this disease; questionable trustworthiness of much information found online; and the decreased ability to filter out unnecessary information and the consequent information overload. Therefore, the pandemic has offered a unique opportunity to study coping with the specific, naturally occurring health-related threat in countries around the world.

Thus far, several studies have investigated cyberchondria during the COVID-19 pandemic. One study has reported that trust in online information and perceived information overload both intensified manifestations of COVID-19-related cyberchondria and suggested "healthy scepticism" about health information and avoidance of information overload as ways of preventing or addressing cyberchondria [39]. This study also found cyberchondria to be a "side effect" of the COVID-19 pandemic. Another study found that during the pandemic, cyberchondria was more prominent among people who used social media as the main source of information about COVID-19 [40], possibly because much information about the pandemic obtained via social media was distorted and untrustworthy [41]. Not surprisingly, cyberchondria was reported to be a risk factor for "coronavirus anxiety" in yet another study conducted during the COVID-19 pandemic, with this anxiety decreasing in the context of being (well) informed about the pandemic [42]. Further studies on cyberchondria during the COVID-19 pandemic are expected to give us a deeper insight into cyberchondria in general.

\section{Prevention and Management of Cyberchondria}

Prevention of cyberchondria does not entail avoidance of OHR because the Internet is the main source of health information in the twenty-first century. As most people who engage in OHR do not develop cyberchondria, this online activity cannot on its own be considered a risk factor for cyberchondria. Therefore, any suggestion to "abstain" from OHR is both unrealistic and misleading.

Prevention efforts need to focus on the way online health information is accessed, interpreted and managed in an effort to improve online health information literacy. The first step is to ensure information is accessed from reliable, reputable and trustworthy sites. Internet users can avail themselves of guides that direct them to such sites and assist them in distinguishing between trustworthy and untrustworthy ones. These guides are usually produced by governments and health or academic organizations and use simple, non-technical language to help individuals with diverse educational backgrounds. It is also important to keep in mind that as valuable as health information may be, it is just that-information. The expectations that Internet users may have of information accessed online need to be adjusted accordingly. That means, for example, that even 
a large quantity and high quality of health information do not necessarily bring a person closer to a diagnosis. If one is after a diagnosis, reliance on "symptom checkers" is problematic [43] and self-diagnosis should be discouraged.

Online health information should empower people so that they are capable of understanding what, if anything, needs to be done about their health at the time the specific enquiry is made. This requires an ability to critically appraise the information. People can also bring that information to an encounter with a healthcare professional, which allows them to be better prepared for the ensuing discussion. When the information causes distress or anxiety and especially in the presence of symptoms, this should probably not be dealt with by further OHR because such coping is likely to induce more distress or anxiety. In other words, seeking further explanation and reassurance via OHR may lead to an interaction between an individual and the Internet in which the individual is likely to feel alone, unsupported, confused and helpless and is therefore likely to "lose". Instead, distressing information needs to be managed via consultation with a healthcare professional, which is an opportunity for any misunderstanding to be clarified and for concerns to hopefully be alleviated or completely quashed.

In the context of prominent cyberchondria, the first task is to ascertain whether there is any associated or underlying psychopathology (e.g. hypochondriasis or OCD). If there is significant associated psychopathology, treating it may alleviate the features of cyberchondria. In other cases, both the cooccurring condition and cyberchondria may need to be targeted in order for cyberchondria to subside. An example of the second approach is a randomized controlled trial evaluating Internet-delivered cognitive-behaviour therapy (CBT) for health anxiety and modified to also target cyberchondria, which demonstrated an improvement in several aspects of cyberchondria, especially excessive OHR, high level of distress and interference with functioning [44••]. Cyberchondriaspecific components incorporated into treatment package included enhancement of online health information literacy, provision of information about helpful and unhelpful patterns of OHR, clarification of the role of (mis)interpretation of online health information in driving further OHR and education about strategies to engage in OHR in a way that would minimize uncertainty and confusion. Furthermore, a decrease in health anxiety was apparently mediated by improvement in cyberchondria.

The cyberchondria-specific modification of CBT used in this study or a similar program might be effective for cyberchondria occurring in different contexts. Such a program might include techniques derived from metacognitive therapy or those based on exposure and response prevention, which is used for OCD. However, no other treatment studies of cyberchondria have been conducted, and as yet, there are no evidence-based treatments for this behavioural and psychopathological constellation.

In the meantime, before the results of controlled treatment studies become available, treatment approaches to cyberchondria might be based on a good understanding of each individual with cyberchondria and the corresponding case formulation. This would allow specific aspects of cyberchondria and specific factors implicated in its maintenance to be targeted. From this perspective, it is important to understand the following:

1) Trigger(s) for OHR, e.g. a symptom that has appeared recently and caused concern;

2) Purpose of OHR, e.g. seeking reassurance about the symptom;

3) Factors that reinforce repeated or persistent OHR, e.g. difficulty in tolerating uncertainty generated by OHR or a belief about being unable to stop OHR;

4) Consequences of cyberchondria, e.g. a conflict with a partner or frequent visits to one's general medical practitioner.

The goal of any therapeutic endeavour in cyberchondria should be to make the person feel confident about their ability to perform OHR. Such confidence would come from the knowledge that the person is able to cope with online health information and its vagaries, i.e. its abundance, inconsistence, contradictory aspects and uncertainty-amplifying potential. The same confidence would make it very unlikely for OHR to be experienced as threatening or overwhelming and to be performed without a sense of control. Ultimately, OHR would feel empowering, not overwhelming.

\section{Conclusion}

Cyberchondria has evolved from being an object of journalistic curiosity to the appropriate focus of a professional interest and scientific enquiry, as reflected in an ever-increasing number of publications devoted to it. Despite this transition, some key questions about cyberchondria remain unanswered. Although its main features have been well described, there is no consensus about its definition and conceptualization. Likewise, theoretical understanding of cyberchondria is yet to be integrated. The status of cyberchondria as a distinct entity or as part of other psychopathology is also unresolved, with some researchers considering it as an epiphenomenon of a "parent" condition, such as hypochondriasis/health anxiety. Not surprisingly, the number of people seeking professional help for cyberchondria as their main complaint or problem seems to be very small. Regardless of these issues, the negative impact of cyberchondria and its public health significance are increasingly recognized but remain to be systematically 
researched. There are various ideas about prevention and management strategies, but these need to be refined and tested. The current situation can therefore be succinctly described as an acceptance that the problem (cyberchondria) exists and that something should be done about it, although its exact nature has not been completely elucidated.

More research is obviously needed to overcome these uncertainties and contradictions about cyberchondria. Future studies need to test the existing and novel theoretical frameworks and conceptualizations and management approaches based on them. Such studies should not only rely on convenience online samples but need to be conducted in clinical populations to establish more clearly the relevance of cyberchondria within the realm of psychopathology.

\section{Compliance with Ethical Standards}

Conflict of Interest Vladan Starcevic, David Berle and Sandra Arnaez each declare no potential conflict of interest.

Human and Animal Rights and Informed Consent This article does not contain any studies with human or animal subjects performed by any of the authors.

\section{References}

Papers of particular interest, published recently, have been highlighted as:

- Of importance

•- Of major importance

1. Starcevic V, Berle D. Cyberchondria: towards a better understanding of excessive health-related Internet use. Exp Rev Neurotherap. 2013;13:205-13. https://doi.org/10.1586/ern.12.162.

2. White RW, Horvitz E. Cyberchondria: studies of the escalation of medical concerns in Web search. ACM Trans Inf Syst. 2009;27(4): 1-37. https://doi.org/10.1145/1629096.1629101.

3. White RW, Horvitz E. Experiences with Web search on medical concerns and self diagnosis. In: Proceedings from the American Medical Informatics Association Annual Symposium 2009 (AMIA 2009). Red Hook: Curran Associates; 2009. p. 696-700.

4. McElroy E, Shevlin M. The development and initial validation of the Cyberchondria Severity Scale (CSS). J Anxiety Disord. 2014;28:259-65. https://doi.org/10.1016/j.janxdis.2013.12.007.

5. Starcevic V. Cyberchondria: challenges of problematic online searches for health-related information. Psychother Psychosom. 2017;86:129-33. https://doi.org/10.1159/000465525.

6.• Starcevic V, Baggio S, Berle D, Khazaal Y, Viswasam K. Cyberchondria and its relationships with related constructs: a network analysis. Psychiatr Q. 2019;90:491-505. https://doi.org/10. 1007/s11126-019-09640-5 Using a network analysis, this study reported that cyberchondria was a relatively distinct entity, with important relationships with problematic Internet use and health anxiety.

7.• Brown RJ, Skelly N, Chew-Graham CA. Online health research and health anxiety: a systematic review and conceptual integration. Clin Psychol Sci Pract. 2020;27:e12299. https://doi.org/10.1111/ cpsp.12299 This is a comprehensive review of the relationship between health anxiety and online health research that also proposes problematic online health research and compulsive online health research as two relatively distinct subtypes of pathological online health research (cyberchondria).

8.• Vismara M, Caricasole V, Starcevic V, Cinosi E, Dell'Osso B, Martinotti G, et al. Is cyberchondria a new transdiagnostic digital compulsive syndrome? A systematic review of the evidence. Compr Psychiatry. 2020;99:152167. https://doi.org/10.1016/j. comppsych.2020.152167 This is a comprehensive review of cyberchondria, with an emphasis on the issues pertaining to definition and conceptualisation. Cyberchondria is construed as a clinically relevant transdiagnostic compulsive behavioural syndrome, closely related to problematic Internet use and usually presenting in association with health anxiety, hypochondriasis and/or obsessive-compulsive disorder.

9. Fergus TA, Dolan SL. Problematic Internet use and Internet searches for medical information: the role of health anxiety. Cyberpsychol Behav Soc Network. 2014;17:761-5. https://doi. org/10.1089/cyber.2014.0169.

10. Singh K, Brown RJ. Health-related Internet habits and health anxiety in university students. Anxiety Stress Coping. 2014;27:54254. https://doi.org/10.1080/10615806.2014.888061.

11. Fergus TA, Spada MM. Cyberchondria: examining relations with problematic Internet use and metacognitive beliefs. Clin Psychol Psychother. 2017;24:1322-30. https://doi.org/10.1002/cpp.2102.

12. Durak-Batigun A, Gor N, Komurcu B, Senkal-Erturk I. Cyberchondria Scale (CS): development, validity and reliability study. Dusunen Adam The Journal of Psychiatry and Neurological Sciences. 2018;31:148-62. https://doi.org/10.5350/ DAJPN2018310203.

13. Selvi Y, Turan SG, Sayin AA, Boysan M, Kandeger A. The Cyberchondria Severity Scale (CSS): validity and reliability study of the Turkish version. Sleep Hypn. 2018;20:241-6. https://doi.org/ 10.5350/Sleep.Hypn.2018.20.0157.

14. Fergus TA, Spada MM. Moving toward a metacognitive conceptualization of cyberchondria: examining the contribution of metacognitive beliefs, beliefs about rituals, and stop signals. J Anxiety Disord. 2018;60:11-9. https://doi.org/10.1016/j.janxdis. 2018.09.003.

15. Marino C, Fergus TA, Vieno A, Bottesi G, Ghisi M, Spada MM. Testing the Italian version of the Cyberchondria Severity Scale and a metacognitive model of cyberchondria. Clin Psychol Psychother. 2020;27:1-16. https://doi.org/10.1002/cpp.2444.

16. Starcevic V. Problematic and compulsive online health research: the two faces of cyberchondria. Clin Psychol Sci Pract. 2020;27: e12320. https://doi.org/10.1111/cpsp.12320.

17. Fergus TA. The Cyberchondria Severity Scale (CSS): an examination of structure and relations with health anxiety in a community sample. J Anxiety Disord. 2014;28:504-10. https://doi.org/10. 1016/j.janxdis.2014.05.006.

18. Fergus TA. Anxiety sensitivity and intolerance of uncertainty as potential risk factors for cyberchondria: a replication and extension examining dimensions of each construct. J Affect Disord. 2015;184:305-9. https://doi.org/10.1016/j.jad.2015.06.017.

19. Norr AM, Albanese BJ, Oglesby ME, Allan NP, Schmidt NB. Anxiety sensitivity and intolerance of uncertainty as potential risk factors for cyberchondria. J Affect Disord. 2015;174:64-9. https:// doi.org/10.1016/j.jad.2014.11.023.

20. Barke A, Bleichhardt G, Rief W, Doering BK. The Cyberchondria Severity Scale (CSS): German validation and development of a short form. Int J Behav Med. 2016;23:595-605. https://doi.org/10. 1007/s12529-016-9549-8.

21. Bajcar B, Babiak J. Self-esteem and cyberchondria: the mediation effects of health anxiety and obsessive-compulsive symptoms in a community sample. Curr Psychol. 2019. https://doi.org/10.1007/ s12144-019-00216-x. 
22. Bajcar B, Babiak J, Olchowska-Kotala A. Cyberchondria and its measurement. The Polish adaptation and psychometric properties of the Cyberchondria Severity Scale CSS-PL. Psychiatr Pol. 2019;53:49-60. https://doi.org/10.12740/PP/81799.

23. Gibler RC, Jastrowski Mano KE, O’Bryan EM, Beadel JR, McLeish AC. The role of pain catastrophizing in cyberchondria among emerging adults. Psychol Health Med. 2019;24:1267-76. https://doi.org/10.1080/13548506.2019.1605087.

24. Jokić-Begić N, Mikac U, Čuržik D, Jokić CS. The development and validation of the Short Cyberchondria Scale (SCS). J Psychopathol Behav Assess. 2019;41:662-76. https://doi.org/10.1007/s10862019-09744-z.

25. McElroy E, Kearney M, Touhey J, Evans J, Cooke Y, Shevlin M. The CSS-12: development and validation of a short-form version of the Cyberchondria Severity Scale. Cyberpsychol Behav Soc Netw. 2019;22:330-5. https://doi.org/10.1089/cyber.2018.0624.

26. González-Rivera JA, Santiago-Olmo KL, Cruz-Rodríguez AS, Pérez-Ojeda RJ, Torres-Cuevas H. Development and validation of the Brief Cyberchondria Scale in Puerto Rico. International Journal of Recent Scientific Research. 2020;11:36734-7. https://doi.org/ 10.24327/ijrsr.2020.1101.4984

27.• Mc Mullan RD, Berle D, Arnáez S, Starcevic V. The relationships between health anxiety, online health information seeking, and cyberchondria: systematic review and meta-analysis. J Affect Disord. 2019;245:270-8. https://doi.org/10.1016/j.jad.2018.11. 037 This systematic review and meta-analysis found a positive correlation between health anxiety and online health research $(r=0.34)$ and an even stronger relationship between health anxiety and cyberchondria $(r=0.62)$.

28. te Poel F, Baumgartner SE, Hartmann T, Tanis M. The curious case of cyberchondria: a longitudinal study on the reciprocal relationship between health anxiety and online health information seeking. J Anxiety Disord. 2016;43:32-40. https://doi.org/10.1016/j.janxdis. 2016.07.009.

29. Fergus TA, Russell LH. Does cyberchondria overlap with health anxiety and obsessive-compulsive symptoms? An examination of latent structure and scale interrelations. J Anxiety Disord. 2016;38: 88-94. https://doi.org/10.1016/j.janxdis.2016.01.009.

30. Zangoulechi Z, Yousefi Z, Keshavarz N. The role of anxiety sensitivity, intolerance of uncertainty, and obsessive-compulsive symptoms in the prediction of cyberchondria. Advances in Bioscience and Clinical Medicine (ABCmed). 2018;6(4):1-6. https://doi.org/10.7575/aiac.abcmed.v.6n.4p.1.

31. Fergus TA. Cyberchondria and intolerance of uncertainty: examining when individuals experience health anxiety in response to internet searches for medical information. Cyberpsychol Behav Soc Network. 2013;16(10):735-9. https://doi.org/10.1089/cyber.2012. 0671 .

32. Mathes BM, Norr AM, Allan NP, Albanese BJ, Schmidt NB. Cyberchondria: overlap with health anxiety and unique relations with impairment, quality of life, and service utilization. Psychiatry Res. 2018;261:204-11.https://doi.org/10.1016/j.psychres.2018.01. 002 .

33. Sommerhalder K, Abraham A, Zufferey MC, Barth J, Abel T. Internet information and medical consultations: experiences from patients' and physicians' perspectives. Patient Educ Couns. 2009;77:266-71. https://doi.org/10.1016/j.pec.2009.03.028.
34. Russ H, Giveon SM, Catarivas MG, Yaphe J. The effect of the Internet on the patient-doctor relationship from the patient's perspective: a survey from primary care. Isr Med Assoc J. 2011;13: $220-4$.

35. Stevenson FA, Kerr C, Murray E, Nazareth I. Information from the Internet and the doctor-patient relationship: the patient perspective - a qualitative study. BMC Fam Pract. 2007;8:47. https://doi.org/ 10.1186/1471-2296-8-47.

36. Wald HS, Dube CE, Anthony DC. Untangling the Web - the impact of Internet use on health care and the physician-patient relationship. Patient Educ Couns. 2007;68:218-24. https://doi.org/10. 1016/j.pec.2007.05.016.

37. Cocco AM, Zordan R, Taylor DMD, Weiland TJ, Dilley SJ, Kant J, et al. Dr Google in the ED: searching for online health information by adult emergency department patients. Med J Aust. 2018;209: 342-7. https://doi.org/10.5694/mja17.00889.

38. Starcevic V, Berle D, Arnáez S, Vismara M, Fineberg NA. The assessment of cyberchondria: instruments for assessing problematic online health-related research. Curr Addict Rep. 2020;7:149-65. https://doi.org/10.1007/s40429-020-00308-w This is the first systematic review of the instruments used for assessment of cyberchondria, providing a critical appraisal of the available tools and suggestions for improving assessment of cyberchondria in the future.

39. Laato S, Najmul Islam AKM, Nazrul Islam M, Whelan E. What drives unverified information sharing and cyberchondria during the COVID-19 pandemic? Eur J Inform Syst. 2020. https://doi.org/10. 1080/0960085X.2020.1770632.

40. Farooq A, Laato S, Najmul Islam AKM. The impact of online information on self-isolation intention during the COVID-19 pandemic: a cross-sectional study. J Med Internet Res. 2020;22: e19128. https://doi.org/10.2196/19128.

41. Kouzy R, Abi Jaoude J, Kraitem A, El Alam MB, Karam B, Adib E, et al. Coronavirus goes viral: quantifying the COVID-19 misinformation epidemic on Twitter. Cureus. 2020;12:e7255. https://doi. org/10.7759/cureus. 7255 .

42. Jungmann SM, Witthöft M. Health anxiety, cyberchondria, and coping in the current COVID-19 pandemic: which factors are related to coronavirus anxiety? J Anxiety Disord. 2020;73:102239. https://doi.org/10.1016/j.janxdis.2020.102239.

43. Hill MG, Sim M, Mills B. The quality of diagnosis and triage advice provided by free online symptom checkers and apps in Australia. Med J Aust. 2020;212:514-9. https://doi.org/10.5694/ mja2.50600.

44.• Newby JM, McElroy E. The impact of Internet-delivered cognitive behavioural therapy for health anxiety on cyberchondria. J Anxiety Disord. 2020;69:102150. https://doi.org/10.1016/j.janxdis.2019. 102150 This was a randomised controlled trial in which Internet-delivered cognitive-behavioural therapy for health anxiety was modified to address cyberchondria and compared to "treatment as usual". Patients who received the former type of treatment showed a significant improvement in cyberchondria.

Publisher's Note Springer Nature remains neutral with regard to jurisdictional claims in published maps and institutional affiliations. 